\title{
Progressive epigenetic dysregulation in neuroendocrine tumour liver metastases
}

\section{Dear Editor,}

The incidence of small intestinal neuroendocrine tumours (SINETs) is increasing and distant metastases are present at diagnosis in $70 \%$ of cases, the liver being the commonest site of metastasis (Yao et al. 2008). Despite this, our understanding of the mechanisms underlying metastatic progression of SINETs is currently limited, and prior studies of the molecular biology of SINET liver metastases (LM) have been performed predominantly in small cohorts utilising candidate-based techniques.

SINETs have a low rate of mutations compared to most cancers. The most frequently mutated gene is CDKN1B (encoding p27, a cell cycle regulator); however, mutations in this gene occur in only $8 \%$ of tumours, and there is no characteristic mutational hotspot (Francis et al. 2013). Furthermore, mutation of CDKN1B does not correlate with the expression of p27 (Crona et al. 2015). We previously identified that SINETs are epigenetically dysregulated, and a panel of candidate driver epimutation genes has been identified (Karpathakis et al. 2016). Therefore, we postulated that metastatic progression in SINETs may also be epigenetically regulated. Here, we present the findings from the largest molecular profiling study of SINET LM performed to date, integrating copy number variance (CNV), DNA methylation, and RNA expression profiling to characterise the mechanisms underlying metastatic progression.

Experimental details of DNA methylation, CNV and RNA expression profiling are as previously published (Karpathakis et al. 2016). Patients provided informed consent for their tissue to be analysed in this study, which was Research Ethics Committee approved (Ref: 09/H0722/27). All cases were reviewed by two expert NET histopathologists (TVL/MN). Nucleic acids were extracted using standard methods (Qiagen, QIAamp DNA Mini kit; Roche, High Pure RNA Paraffin kit). H\&E-stained sections were evaluated to ensure $>80 \%$ purity of tumour specimens. Methylation profiling was performed on the HumanMethylation450 BeadChip (HM450) (Illumina). Methylation data were analysed using ChAMP pipeline (https://www.bioconductor.org/packages/release/bioc/ html/ChAMP.html). Whole-genome methylation profiling using methylated DNA immunoprecipitation sequencing (MeDIP) was performed as previously described. MeDIP data were analysed using the custom pipeline MeDUSA, v2.0 (https://www.ucl.ac.uk/cancer/research/departmentcancer-biology/medical-genomics-group/past-projects/ medusa-project). Gene expression levels were quantified using the whole genome cDNA-mediated annealing, selection and ligation (DASL) (Illumina) assay. Expression data were analysed using the 'LIMMA' package in $\mathrm{R}$ (https://bioconductor.org/packages/release/bioc/html/ limma.html). Raw data from this study has previously been deposited in GEO (Accession number: GSE73832) (Karpathakis et al. 2016).

In summary, $n=90$ samples underwent array-based DNA methylation analysis, $n=26$ samples underwent methylation-specific immunoprecipitation followed by DNA sequencing and $n=49$ underwent array-based RNA expression analysis. Of cases with relevant clinical data, $93 \%$ had received no systemic treatment prior to specimen collection (27/29 cases).

The CNV profile of SINET LM $(n=20)$ mirrors that of primary tumours with the most frequent alteration of chr18 LOH seen in 79\% of cases. A greater proportion of LM demonstrates amplification of chr20 (42\%) and deletion of chr19 (35\%), whereas gain of $17 \mathrm{q}$ is found only in LM (21\%). A trend of increased incidence of CNVs was seen in LM compared to that of SINET primary tumours (SINET primary: median $78 \mathrm{mbp}$; LM: median $114 \mathrm{mbp}, P=0.08)$.

Comparison of methylation profiles of SINET LM to those of primary SINETs identified 29,263 methylation 
Table 1 Methylation and expression profile of SINET LM demonstrates progressive dysregulation compared to SINET primary tumours in a panel of genes $(P<0.05$ in bold).

\begin{tabular}{|c|c|c|c|c|c|c|c|c|c|c|}
\hline & \multicolumn{5}{|c|}{ Median methylation } & \multicolumn{5}{|c|}{ Median expression } \\
\hline & NSI & SINET & LM & $\begin{array}{l}\text { LM } \\
\text { progressing } \\
\text { trend? }\end{array}$ & $\begin{array}{c}P \text { (LM vs } \\
\text { SINET) }\end{array}$ & NSI & SINET & LM & $\begin{array}{l}\text { LM } \\
\text { progressing } \\
\text { trend? }\end{array}$ & $\begin{array}{c}P \text { (LM vs } \\
\text { SINET) }\end{array}$ \\
\hline \multicolumn{11}{|c|}{$\overline{\text { Downregulated }}$} \\
\hline $\mathrm{CDX} 1$ & 0.55 & 0.90 & 0.91 & Yes & 0.48 & 5481.4 & 1503.0 & 570.8 & Yes & 0.03 \\
\hline FBP1 & 0.43 & 0.82 & 0.83 & Yes & 0.58 & $10,896.0$ & 2193.1 & 2937.5 & No & 0.54 \\
\hline TMEM171 & 0.18 & 0.61 & 0.73 & Yes & 0.11 & 3632.7 & 519.6 & 201.9 & Yes & 0.08 \\
\hline C20orf54 & 0.34 & 0.64 & 0.60 & No & 0.64 & 2320.5 & 577.0 & 176.7 & Yes & 0.056 \\
\hline GATA5 & 0.89 & 0.69 & 0.57 & Yes & 0.006 & 658.2 & 64.0 & 65.8 & No & 0.96 \\
\hline NGEF & 0.89 & 0.72 & 0.60 & Yes & 0.018 & 3819.7 & 512.2 & 309.9 & Yes & 0.23 \\
\hline PNLIPRP2 & 0.77 & 0.47 & 0.33 & Yes & 0.007 & 1419.4 & 235.0 & 95.5 & Yes & 0.34 \\
\hline TRIM15 & 0.53 & 0.87 & 0.87 & No & 0.65 & 615.8 & 93.1 & 27.2 & Yes & 0.009 \\
\hline \multicolumn{11}{|l|}{ Upregulated } \\
\hline PTPRN & 0.38 & 0.11 & 0.07 & Yes & 0.001 & 349.8 & 4319.1 & 5456.3 & Yes & 0.1 \\
\hline C3orf14 & 0.32 & 0.12 & 0.07 & Yes & $<0.001$ & 212.4 & 1016.7 & 1803.9 & Yes & 0.002 \\
\hline CNTNAP5 & 0.26 & 0.09 & 0.08 & Yes & 0.009 & 280.3 & 4343.1 & 3518.3 & No & 0.22 \\
\hline DSCAM & 0.50 & 0.15 & 0.08 & Yes & $<0.001$ & 59.9 & 1142.3 & 1028.6 & No & 0.61 \\
\hline GDAP1L1 & 0.38 & 0.08 & 0.05 & Yes & 0.002 & 74.3 & 659.7 & 1051.8 & Yes & 0.07 \\
\hline PCSK1 & 0.38 & 0.08 & 0.05 & Yes & 0.001 & 217.9 & 2420.4 & 2911.0 & Yes & 0.39 \\
\hline PRLHR & 0.44 & 0.13 & 0.06 & Yes & $<0.001$ & 193.6 & 3909.3 & 2676.2 & No & 0.08 \\
\hline SNTG1 & 0.28 & 0.07 & 0.04 & Yes & $<0.001$ & 78.6 & 2126.9 & 3396.3 & Yes & 0.003 \\
\hline CELSR3 & 0.10 & 0.57 & 0.67 & Yes & 0.11 & 2029.6 & 7869.0 & 9442.6 & Yes & 0.17 \\
\hline GIPR & 0.30 & 0.66 & 0.74 & Yes & 0.034 & 248.1 & 1900.0 & 2283.6 & Yes & 0.27 \\
\hline KCNH6 & 0.07 & 0.36 & 0.48 & Yes & 0.01 & 998.3 & 5146.7 & 5935.3 & Yes & 0.35 \\
\hline LMX1B & 0.30 & 0.59 & 0.60 & Yes & 0.99 & 286.8 & 3503.9 & 1979.4 & No & 0.034 \\
\hline RUNDC3A & 0.08 & 0.42 & 0.66 & Yes & 0.02 & 454.2 & 3966.4 & 5677.8 & Yes & 0.081 \\
\hline
\end{tabular}

LM, liver metastasis; NSI, normal small intestine; SINET, small intestinal primary neuroendocrine tumour.

variable positions (MVPs) (adj $P<0.05$ ). Using a cutoff of $>30 \%$ difference in methylation between SINET primaries and LM, MVPs involving eight genes were identified (CLEC16A, HOXC4, HOXD4, IGF2AS, INS-IGF2, LDHA, RTN4RL1 and SASH1). This suggests that the methylation profile of SINET primaries and metastases are broadly similar and that these epigenetic differences occurring in metastatic progression may be more subtle than those involved in primary tumourigenesis. Global hypomethylation is noted in SINET primary tumours and occurs to an even greater extent in SINET LM (normal tissue methylation 0.628 , primary 0.572 , LM 0.515 , $P<0.001$ ).

Almost $3000 \quad(n=2857)$ genes were significantly differentially expressed between LM and primary tumours. Using a $>3$-fold alteration in gene expression, more genes were found to be upregulated $(n=321)$ than downregulated $(n=171)$ in LM. KEGG pathway analysis (http://www.webgestalt.org) of differentially expressed genes between LM and SINET primary identified significant enrichment of multiple cancerrelated pathways overexpressed in LM including PI3K signalling events, ErbB1 downstream signalling,
PDGFR $\beta$ signalling pathway and mTOR signalling pathway (adjusted $P<0.001$ ).

Analysis of SINET LM identified progressive changes between SINET primaries and LM in DNA methylation and RNA expression in genes that had previously been identified in primary tumours when compared to normal tissue. This phenomenon was observed in a panel of 21 epigenetically dysregulated candidate driver genes that were previously identified (Karpathakis et al. 2016) (Table 1).

LM demonstrated hyper/hypomethylation of all 21 genes in concordance with the pattern seen in SINET primaries when compared to normal tissue. In 19 genes (90.5\% of the panel), a trend for progressive hyper/hypomethylation was demonstrated in LM, of which 14 genes $(66.6 \%)$ of the panel were significantly differentially methylated compared to the primary SINET.

All the 21 genes included in the panel demonstrated over/under expression in LM in concordance with SINET primary tumours. In 15 genes $(71.4 \%$ of panel), there was a progression of aberrant expression to a greater extent in LM than was demonstrated in SINET primaries (Fig. 1 and Table 1).

Published by Bioscientifica Ltd. 


\section{Methylation}

Downregulated
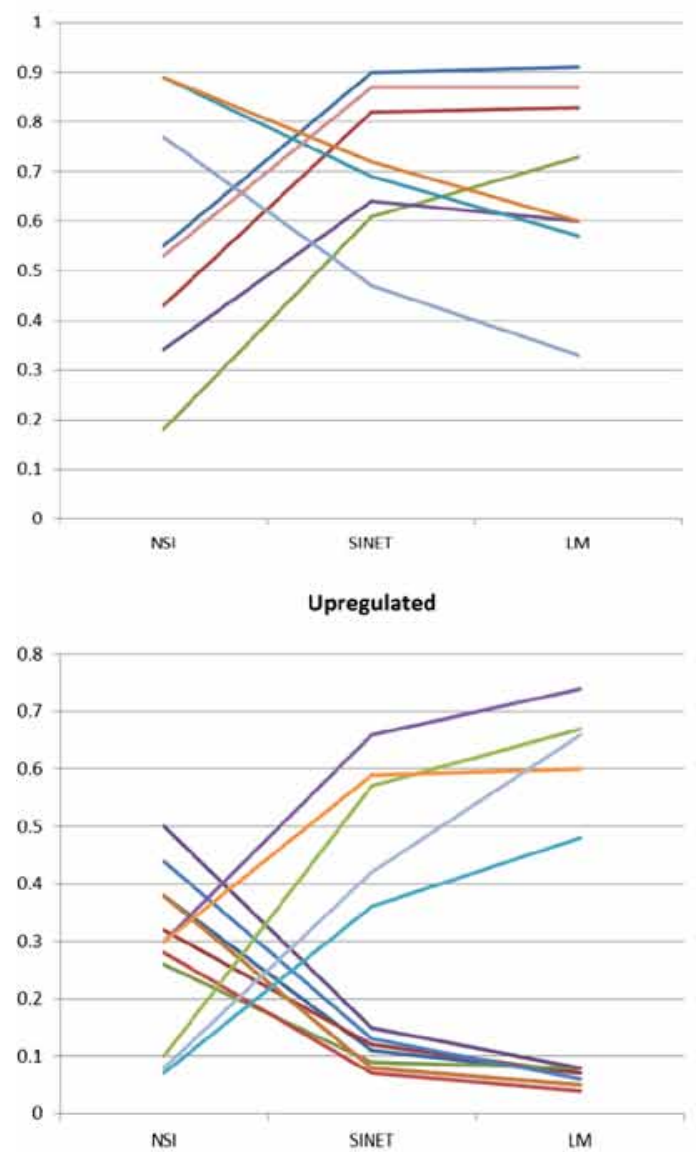

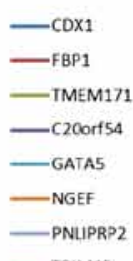

- TRIM15

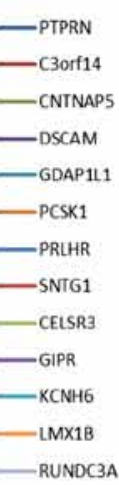

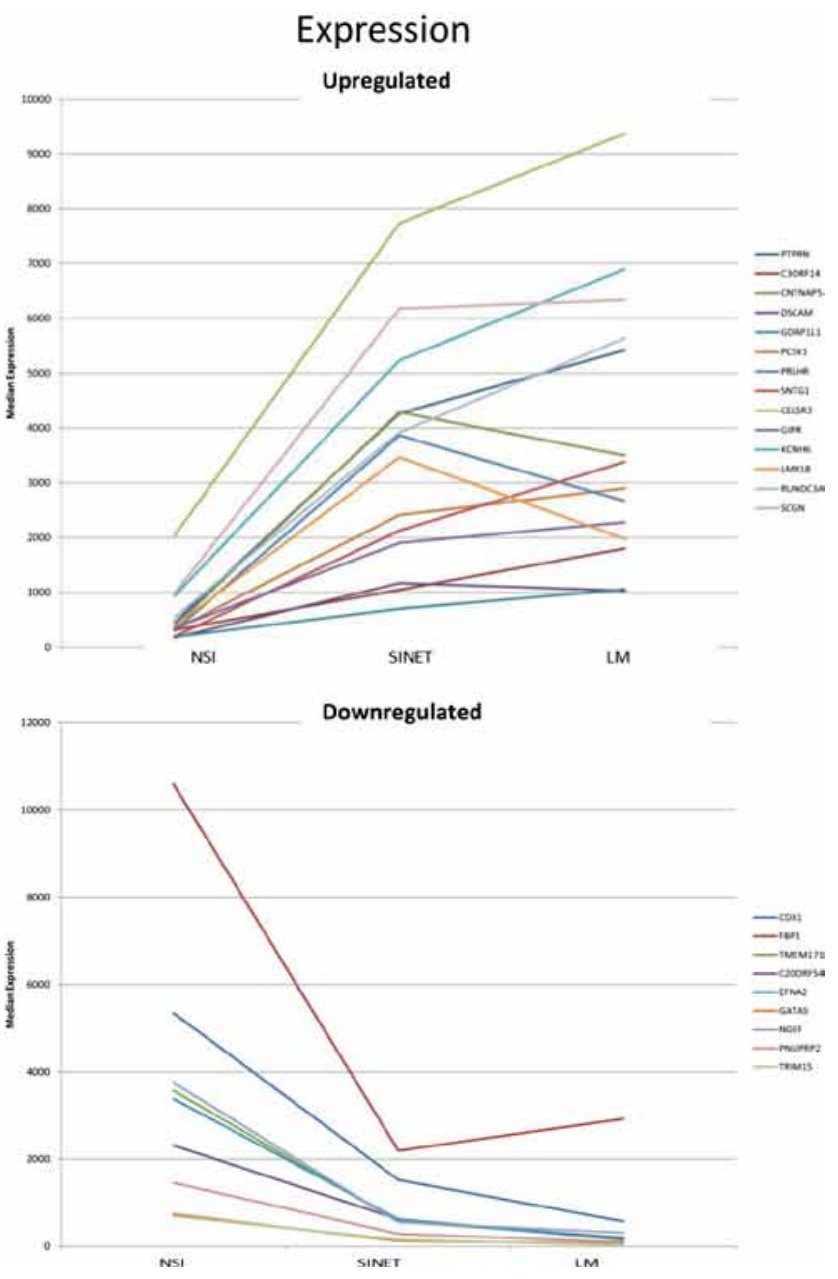

Figure 1

Methylation and expression profile of SINET LM demonstrates progressive dysregulation compared to SINET primary tumours in a panel of genes.

Validation of SINET LM methylation status was done in an independent cohort of seven LM profiled by methylated DNA immunoprecipitation sequencing (MeDIP-seq). It was demonstrated that 20/21 (95.2\%) genes exhibited concordant trends in methylation during progression from NSI to SI primary tumour to liver metastasis as was identified using HM450 profiling. Statistically significant progressive aberrant methylation was demonstrated in 10/21 genes (47.6\%).

Validation of SINET LM expression status was performed utilising a publicly available dataset including three SINET primaries and three LM (GSE9576, Leja et al. 2009). In total, $10 / 21(47.6 \%)$ of the candidate panel demonstrated a trend for progressive dysregulation in LM compared to primary tumours in keeping with the findings from the discovery dataset. The small number of cases included in this validation set limits the ability to confirm the statistical significance.
Through integrated DNA methylation, CNV and RNA expression analysis, we have identified progressive genomic derangements in SINET LM when compared to those in primary tumours.

CNVs were seen more frequently in LM, in particular arm-level amplifications, as previously reported (Hashemi et al. 2013). Amplification of chromosome $17 \mathrm{q}$ was observed more frequently in LM than that in primaries in this cohort. This alteration has previously been described in both SINET and pancreatic NET primary tumours, but this is the first time that increased frequency in LM has been identified. Chromosome 17 harbours the proto-oncogene HER2/neu (17q11-21), amplification of which may be related to a more aggressive phenotype.

The finding of progressive global hypomethylation in metastases compared to primary tumours is in keeping with previously published data (Verdugo et al. 2014). A pattern of progressive aberrant methylation observed in

Published by Bioscientifica Ltd. 
our previously identified panel of 21 epimutated genes in liver metastases suggests that increasing epigenetic dysregulation may drive progression to metastasis.

Transcriptome profiling demonstrated differential expression of 492 genes between LM and SINET primary tumours, which may represent drivers of metastasis, including components of the PI3K/mTOR pathways. Progressively dysregulated expression of the panel of candidate genes was demonstrated in liver metastases compared to that in primary tumours. This indicates that deregulation of pathways associated with development of SINET primary tumours occurs to a greater extent during metastatic progression.

In total, $71.4 \%(15 / 21$, expression) to $90.5 \%$ (19/21, methylation) of cases are affected by progressive dysregulation in association with metastasis. The gene encoding the gastric inhibitory polypeptide receptor (GIPR) is one of a panel of epigenetically dysregulated genes in SINETs which is significantly progressively hypermethylated in LM compared to primary tumours. This may represent a target for novel therapeutic agents in the management of SINETs and has already been investigated as a target for novel imaging modalities (Sherman et al. 2013).

In summary, integrated genomic analysis of a large cohort of SINET LM has identified novel molecular mechanisms associated with metastatic progression. Epigenetic dysregulation of a panel of 21 candidate genes was identified in LM concordant with those found in primary SINET. Components of cancer-related pathways including PI3K, mTOR and ErbB1 are overexpressed in liver metastases compared to normal tissue, which may be utilised as therapeutic targets. Current clinical practice includes the use of agents targeting the mTOR pathway is based on evidence from the RADIANT trials of everolimus in pancreatic and SINETs (Yao et al. 2016). The use of second-line dual mTORC/PI3K inhibition for pancreatic NETs was not supported in a recent clinical trial (Fazio et al. 2016). Our data suggest that the development of novel agents targeting epigenetic modifications in these pathways may hinder metastatic progression.

Large-scale alterations in the transcriptome of SINET LM compared to primary tumours have been identified, with more subtle alterations in the methylome. This may indicate that small alterations in the epigenetic status of key genes are sufficient to drive metastatic progression or that alternative mechanisms are also contributing to progression including for example histone modifications. To date, there have been no identified driver genetic mutations responsible for SINET development or progression. The data presented in this manuscript suggest that epigenetic alterations are significant in this tumour type. We believe that future research should be focused on further elucidating epigenetic mechanisms in the evolution of neuroendocrine tumours.

Anna Karpathakis ${ }^{1,2}$ Harpreet Dibra ${ }^{1}$ Christodoulos Pipinikas ${ }^{1}$ Andrew Feber ${ }^{1}$ Tiffany Morris ${ }^{1}$ Joshua Francis ${ }^{3}$

Dahmane Oukrif ${ }^{1}$

Dalvinder Mandair ${ }^{1,2}$

Marinos Pericleous ${ }^{2}$

Mullan Mohmaduvesh ${ }^{2}$

Stefano Serra ${ }^{4}$

Olagunju Ogunbiyi ${ }^{2}$

Marco Novelli ${ }^{1}$

TuVinh Luong ${ }^{2}$

Sylvia L Asa ${ }^{4}$

Matthew Kulkes

Christos Toumpanakis ${ }^{2}$

Tim Meyer ${ }^{1,2}$

Martyn Caplin ${ }^{2}$

Stephan Beck ${ }^{1}$

Christina Thirlwell ${ }^{1,2}$

${ }^{1}$ University College London, London, UK

${ }^{2}$ The Royal Free Hospital, London, UK

${ }^{3}$ The Broad Institute, Boston, Massachusetts, USA

${ }^{4}$ UHN Princess Margaret Cancer Centre, Toronto,

Ontario, Canada

${ }^{5}$ DanaFaber Cancer Institute, Boston, Massachusetts, USA

(Correspondence should be addressed to C Thirlwell; email: christina.thirlwell@ucl.ac.uk)

Declaration of interest

The authors declare that there is no conflict of interest that could be perceived as prejudicing the impartiality of the research reported.

\section{Funding}

This work was supported by the UCL Experimental Cancer Medicine Centre, NIHR UCLH Biomedical Research Centre, Cancer Research UK, Medical Research Council and The Wellcome Trust. 


\section{References}

Crona J, Gustavsson T, Norlen O, Edfeldt K, Akerstrom T, Westin G, Hellman P, Bjorklund P \& Stalberg P 2015 Somatic mutations and genetic heterogeneity at the CDKN1B locus in small intestinal neuroendocrine tumors. Annals of Surgical Oncology 22 S1428-S1435. (doi:10.1245/s10434-014-4351-9)

Fazio N, Buzzoni R, Baudin E, Antonuzzo L, Hubner R, Lahner H, De Herder W, Raderer M, Teule A, Capdevila J, et al. 2016 A phase II study of BEZ235 in patients with everolimus resistant advanced pancreatic neuroendcrine tumours. Anticancer Research 36 11-21.

Francis JM, Kiezun A, Ramos AH, Serra S, Pedamallu CS, Qian ZR, Banck MS, Kanwar R, Kulkarni AA, Karpathakis A, et al. 2013 Somatic mutation of CDKN1B in small intestine neuroendocrine tumors. Nature Genetics 45 713-719. (doi:10.1038/ng.2821)

Hashemi J, Fotouhi O, Sulaiman L, Kjellman M, Hoog A, Zedenius J \& Larsson C 2013 Copy number alterations in small intestinal neuroendocrine tumors determined by array comparative genomic hybridization. BMC Cancer 13 505. (doi:10.1186/1471-2407-13-505)

Karpathakis A, Dibra H, Pipinikas C, Feber A, Morris T, Francis J, Oukrif D, Mandair D, Pericleous M, Mohmaduvesh M, et al. 2016 Prognostic impact of novel molecular subtypes of small intestinal neuroendocrine tumours. Clinical Cancer Research 22 1483-1486. (doi:10.1158/1078-0432.CCR-15-0373)
Leja J, Essaghir A, Essand M, Wester K, Oberg K, Totterman TH, Lloyd R, Vasmatzis G, Demoulin JB \& Giandomenico V 2009 Novel markers for enterochromaffin cells and gastrointestinal neuroendocrine carcinomas. Modern Pathology 22 250-258. (doi:10.1038/ modpathol.2008.174)

Sherman SK, Carr JC, Wang D, O'Dorisio MS, O'Dorisio TM \& Howe JR 2013 Gastric inhibitory polypeptide receptor (GIPR) is a promising target for imaging and therapy in neuroendocrine tumors. Surgery 154 261-272; discussion 1214. (doi:10.1016/j.surg.2013.04.052)

Verdugo AD, Crona J, Starker L, Stalberg P, Akerstrom G, Westin G, Hellman P \& Bjorklund P 2014 Global DNA methylation patterns through an array-based approach in small intestinal neuroendocrine tumors. Endocrine-Related Cancer 21 L5-L7. (doi:10.1530/ERC-13-0481)

Yao JC, Hassan M, Phan A, Dagohoy C, Leary C, Mares JE, Abdalla EK, Fleming JB, Vauthey JN, Rashid A, et al. 2008 One hundred years after 'carcinoid': epidemiology of and prognostic factors for neuroendocrine tumors in 35,825 cases in the United States. Journal of Clinical Oncology 26 968-977. (doi:10.1200/JCO.2007.15.4377)

Yao JC, Fazio N, Singh S, Buzzoni R, Carnaghi C, Wolin E, Tomasek J, Raderer M, Lahner H, Voi M, et al. 2016 Everolimus for the treatment of advanced non-functional neuroendcrine tumours of the lung or gastrointestinal tract (RADIANT-4): a randomised placebo controlled phase 3 study. Lancet 387 1206-1213. (doi:10.1016/ S0140-6736(15)00817-X)

Received in final form 17 December 2016

Accepted 20 December 2016

Accepted Preprint published online 20 December 2016
(C) 2017 Society for Endocrinology Printed in Great Britain
Published by Bioscientifica Ltd. 